\title{
ESTUDIOS SOBRE EL DIÁLOGO RENACENTISTA DESDE UNA PERSPECTIVA EUROPEA (1898-2005). $\mathrm{I}^{1}$
}

JORGE LEDO

Centre for Modern Thought

University of Aberdeen

\section{RESUMEN}

Este artículo es una recopilación crítica de los principales trabajos que sobre el diálogo renacentista se han publicado entre 1898 y 2005 en lengua italiana, inglesa y francesa. Al tratarse de un arco temporal amplio, he intentado ofrecer al lector una narrativa coherente de las líneas que han orientado la investigación durante el siglo pasado, estableciendo puntos de contacto entre dominios lingüísticos y acercamientos críticos que no siempre son evidentes sin una revisión comprehensiva de la bibliografía. La razón para limitar la selección de las obras a tres dominios idiomáticos — con alguna breve incursión en el territorio alemánviene definida por las capacidades comparativas del corpus y por el marco crítico que pueden ofrecer para la adscripción —crítica o no- de otros dominios lingüísticos a la narrativa que, creo, ofrecen. Queda para una mejor ocasión una recopilación de similares características en torno al diálogo renacentista español.

Palabras clave: diálogo, Renacimiento, Italia, Francia, Inglaterra, literatura, literatura comparada, crítica literaria

\section{STUDIES ON RENAISSANCE DIALOGUE FROM AN EUROPEAN PERSPECTIVE (1898-005)}

\section{ABTRACT}

This essay is a comprehensive review of the main critical works on Renaissance dialogue published in Italian, French and English between 1898 and 2005. Due to the temporal and linguistic extension of the topic, I have drawn - when possible - a narrative from the different critical developments, offering to the reader connections between separate linguistic domains and discrete critical approaches. These links are not always evident without an exhaustive scrutiny of the bibliography. The limiting of my selection to three languages - with a few references to the German realm - is motivated by the comparative possibilities and the critical

${ }^{1}$ La primera versión de este trabajo fue redactada en 2005 gracias a una beca de Formación de Profesorado Universitario (M. E. C.), agradezco las observaciones que María José Vega, Emilio Blanco y Josep Solervicens hicieron en su momento al texto. Las revisiones, adiciones y cambios posteriores no podrían haber sido realizados sin el apoyo económico e institucional del Centre for Modern Thought (University of Aberdeen). 
framework it offers. In the near future I will hopefully write a review of the studies on Spanish Renaissance dialogue.

Keywords: Dialogue, Renaissance, Italy, France, England, Literature, Comparative literature, Literary studies.

\begin{abstract}
He querido ofrecer en estas páginas las líneas evolutivas que el estudio del diálogo renacentista ha seguido durante el siglo pasado. La limitación para el lector avisado será evidente: existe una clara primacía del diálogo italiano y, ante todo, de un corpus restringido del mismo. Se trata, me temo, del principal problema que presenta la bibliografía secundaria cuando se pretende realizar un estudio comparado del género durante el Renacimiento. Componer una narrativa de estas características me ha forzado a prescindir de los estudios sobre el diálogo renacentista castellano y catalán, del trabajo de críticos e historiadores alemanes -Klaus Hempfer (2002, 2004 y 2005), Roger Friedlein (2004 y 2005), entre otros-, de importantes monografías sobre tipologías concretas y de obras de conjunto como las de David Marsh (1980) y Leonid M. Batkin (1990), sin duda esta última la mejor síntesis -y la más injustamente olvidada-sobre la cultura del diálogo humanista italiano. Dejo para una mejor y pronta ocasión la segunda parte de esta reseña: una breve historia de todos ellos y de las causas históricas, críticas y literarias para su ubicación en una narrativa diferenciada.
\end{abstract}

El estudio diacrónico del diálogo como manifestación literaria pertenece con todo derecho al siglo Xx. Durante estos últimos cien años, se han realizado varios intentos aproximativos al género que partían de la finalidad y la tópica, pasando por los métodos compositivos, las fuentes y los principios retóricos y dialécticos implicados en su creación o en la cultura que los gestó, hasta llegar al estudio y a la contextualización de las ideas teóricas que sobre el mismo había generado la tradición poética occidental. Esta última percepción es la que ha acabado por imponerse en los últimos años, con especial atención al diálogo como forma poética que surgió y pasó a formar parte integrante del pensamiento literario y de la labor intelectual durante el Renacimiento. Las intuiciones que rondan a la forma durante el siglo XVI son consecuencia no sólo del nuevo "método especulativo" poético que acabará desembocando en la Teoría Literaria de los curricula actuales, sino también - y principalmente- de la dificultad de absorción de nuevas formas dentro del pensamiento poético. El lector se encontrará en estas páginas con un recorrido histórico por los estudios sobre el diálogo renacentista y su poética a lo largo del siglo XX. Para abarcar su variedad, separaré artificialmente aquellos que han atendido al cultivo del género de los que se han ocupado de su poética. En tal distinción se ejerce un cierto grado de violencia interpretativa, ya que al menos desde la monografía de Kenneth J. Wilson, Incomplete Fictions. The Formation of English Renaissance Dialogue (1985) se han venido realizando síntesis históricas que intentan combinar las observaciones teóricas del periodo con su práctica literaria. 
Los estudios sobre la producción de diálogos cuenta, en realidad, con mayor tradición histórica. La obra fundadora en esta dirección es la amplia monografía de Rudolf Hirzel, Der Dialog. Ein literarhistorischer Versuch ([1895] 1963). El estudio de Hirzel, desde el punto de vista de Franco Pignatti (1999: 408), «trata de establecer las líneas con que componer una historia del diálogo a través de las distintas épocas de la literatura occidental con una marcada asimetría en favor de las literaturas antiguas.» Además, este primer trabajo está claramente marcado por la concepción vigente en el comparatismo alemán de principios de siglo y de amplia influencia durante al menos los cincuenta años siguientes: la idea de una "evolución" de las obras literarias gracias a la implicación plena y unívoca en su desarrollo de obras anteriores transformadas por el "genio". La importancia de la obra, más allá de la metodología y de la poca atención que presta al diálogo renacentista —apenas cincuenta de las mil páginas que contienen sus dos volúmenes-, fue mucha, puesto que cimentó y estructuró los pilares en el estudio del diálogo clásico, generando la concepción tripartita entre diálogos lucianescos, ciceronianos y platónicos que perdura hasta nuestros días.

El primer trabajo en valerse de este distingo fue la monografía de Elizabeth Merrill: The Dialogue in English Literature ([1911] 1969), que conjuga de un modo un tanto confuso la división en grandes estructuras o modelos dialógicos y el estudio histórico de los mismos. Así, su texto está dividido en dos capítulos-marco que versan sobre el diálogo en la Edad Media y su influencia en el siglo XVI (capítulo II) y el diálogo en el siglo XIX (capítulo VI), mientras que los tres capítulos que abrazan se encargan respectivamente de tres categorías distintas que corresponden con un modelo clásico: el diálogo polémico (cuya fuente principal es Luciano), el diálogo expositivo (Cicerón) y el diálogo filosófico (obviamente tras la estela platónica). La impresión que permanece tras recorrer el texto de Merrill es la de hallarse ante un loable intento de síntesis marcado por la obviedad de sus fuentes cuando no se trata de obras inglesas, cuya selección, por otra parte, es muy parcial. En este sentido, su trabajo se diseña como un manual e intenta establecer un conjunto de líneas principales a las que adaptar todo el corpus de diálogos de producción inglesa.

Algunos de los hallazgos de Merrill serán retomados y se harán tópicos en los estudios sobre el género, por ejemplo, la concepción de que la amplia mayoría de los diálogos renacentistas reflejan el peso de las formas excesivamente simplificadas y doctrinales del debate medieval -idea que ella misma matiza mediante otra interpretación que se hará no menos tópica, a saber, la concepción del diálogo renacentista como diálogo realista, con una voluntad de historicidad y de recreación de las costumbres y de las formas de toda una época- ya se encuentra en Bildung und Wissenschaft im Zeitalter der Renaissance in Italien de Leonardo Olschki aplicado a ejemplos exclusivamente italianos, como recuerda Pignatti (1999: 409). 
La primera mitad del siglo se cierra con la tesis de Giovanna Wyss Morigi: Contributo allo studio del dialogo all'epoca dell'Umanesimo e del Rinascimento (1947), que parte de la consideración del diálogo como género mixto «capace di accogliere in sè elementi di dottrina ed elementi di fantasia. Esso è per eccellenza il campo in cui si esplica l'attività logicoragionatrice, senza rifiutare il diritto di cittadinanza alla retorica e nemmeno agli accorgimenti dell'arte drammatica» (p. 17). Tras esta premisa, y una síntesis excesiva del peso del diálogo clásico en el diálogo renacentista, el estudio se divide en tres partes que corresponden para su autora con las tres etapas de desarrollo de este último: el siglo XIV, representado únicamente por Petrarca y concebido como pre-humanista; el siglo XV, donde el diálogo se identifica de manera consciente con las figuras de Valla, Bracciolini, Pontano y Alberti; y el siglo XVI, caracterizado por una «marcata independenza dai modelli classici» (p. 18).

Junto a esta evolución diacrónica, una de las propuestas más discutidas - en especial por Giulio Ferroni- del Contributo ha sido la organización de los diálogos renacentistas a partir de la finalidad perseguida por sus autores, de manera que cabe hablar de dialoghi insegnativi, que se dividen en diálogos primordialmente didácticos y en didáctico-polémicos, y de dialoghi piacevole, que contienen tres variedades principales: representación de ambientes, ejercicios satíricos y diálogos afines a la comedia.

La segunda mitad del siglo se caracterizó por un ahondamiento mucho mayor en la naturaleza histórica, literaria y poética del género. Comienzan a entrar en juego nuevos elementos que trazan algunas líneas de investigación todavía abiertas en la actualidad. Por ejemplo, el magistral estudio de Cesare Vasoli La dialettica e la retorica dell'Umanesimo. «Invenzione»e «metodo» nella cultura del XV e XVI secolo (1968) señala de una manera coherente y sistemática la progresiva desvinculación por parte de los humanistas de la lógica escolástica a favor de un género argumentativo más retorizado, esto es, mucho más sujeto a estructuras ficcionales, ejemplares y ante todo pragmáticas. En cierta medida, es este giro que Vasoli fundamenta en la figura de Valla el que convierte al diálogo humanista en una variante fijada del género $\mathrm{y}$, ante todo, tal consideración abre la perspectiva para entender la base dialógica como pilar fundamental de la ficción moderna.

En la misma década surge el primer intento de sistematización y de análisis comprehensivo del diálogo cuatrocentista de la pluma de uno de los principales estudiosos italianos de la época; me refiero a Francesco Tateo, que en la segunda parte de su Tradizione e realtà nell'Umanesimo italiano (1967) recorre la vía señalada por Vasoli al considerar los aspectos esencialmente teatrales y antitéticos del modelo ciceroniano en el diálogo latino del periodo. La tesis principal de Tateo bascula sobre el modelo ciceroniano, que deviene el punto de partida - tanto por identificación 
como por alejamiento- para analizar la mayor parte de dialoguistas cuatrocentistas de renombre. El ejemplo más patente en la tesis de Tateo encarna de nuevo en la figura de Lorenzo Valla y de dos de sus diálogos: De voluptate y De professione religiosorum.

Las apreciaciones contenidas en los trabajos de Vasoli y Tateo, y la ampliación de miras que supusieron con respecto a los estudios de Wyss Morigi o de Merrill, condujeron a la introducción de la retórica y de la dialéctica en el estudio del género desde una perspectiva histórica. Esta nueva constelación de problemas fue tratada durante la década de los setenta en tres artículos fundamentales para el examen de la materia: los dos trabajos de Luisa Mulas (1980: 97-117, 1982: 245-263), muy influidos por el Traité de l'argumentation de Perelman y Olbretchs-Tyteca, defendían ante todo la construcción retórica de la argumentación en el diálogo renacentista y, por tanto, su inconsistencia lógica. En el otro extremo se encuentra el trabajo de C. J. R. Armstrong (1976: 36-51), que se había propuesto destacar cómo desde una perspectiva teórica la dialéctica era una disciplina esencial en la construcción del género. En realidad, durante los veinticinco años subsiguientes, los estudios sobre el diálogo se decantarían — con diversa intensidad - por uno u otro itinerario, debido a la excesiva atención prestada a los tratados específicos sobre ambas artes y no al conjunto general de los textos teóricos que los pudieran determinar.

La primera tentativa de analizar el género desde premisas poéticas renacentistas y desde la producción literaria de la época partió, de nuevo, del diálogo inglés. En Incomplete Fictions. The Formation of English Renaissance Dialogue (1985), Kenneth J. Wilson intenta conjugar la búsqueda de las raíces históricas y teóricas y la producción de tres dialoguistas ingleses de primer orden (Elyot, Ascham y Moro) para hallar las motivaciones de la tardanza del diálogo en hallar su estatuto como categoría literaria. La tesis principal del inglés es que sus dificultades adscriptivas se deben a su complejidad y a la vinculación de una cantidad enorme de conceptos, temáticas y variedades que lo convierten en un mediate art.

Tres años más tarde aparece un artículo determinante en nuestro campo de estudio: «Il diálogo cinquecentesco italiano tra diegesi e mimesi» (1988), posteriormente titulado «Teoria e 'situazione' del dialogo nel Cinquecento Italiano» (1990), de Nuccio Ordine. Para el italiano, siguiendo una de las líneas directrices de los estudios de Bernard Weinberg, el debate teórico acerca del diálogo en el Cinquecento se articula en torno a cuestiones de retórica y poética. La retórica coincide en todos los tratados específicos sobre el género (Sigonio, Speroni y Tasso) con el aislamiento del género a la doxa a partir de la conocida oposición aristotélica entre opinión y verdad. Por otra parte, esta primacía de la opinio supone la asimilación del diálogo con el género epidíctico dentro de los tres genera causarum de la retórica clásica, identificación que en el caso 
de Speroni establece a su vez un vínculo estrecho entre la elocutio y el delectare. La cuestión poética principal reside para Ordine en la división aristotélico-platónica entre género narrativo o diegético, representativo o mimético y mixto. El predominio de una u otra especie le sirve para establecer la línea evolutiva del diálogo quinientista. Así, el diálogo diegético - con una figura autorial que introduce las interlocuciones de sus personajes y su circunstancia- está perfectamente representado en los dos principales diálogos cortesanos del siglo XVI italiano: Gli Asolani (1505) de Pietro Bembo y el Cortegiano (1528) de Baldassare Castiglione, puesto que en ambos «abbiamo riscontrato un'importante omologia tra luoghi, circostanze e personaggi» (p. 17) y «non presentano affanno si percorsi di ricerca della verità: il loro compito è quello di esaltare valori già condivisi dalla comunità a cui si rivolgono, per accrescere ancora di più attorno ad essi l'adesione e il consenso» (p. 20). El ejemplo inverso de estos prototipos lo ofrece el Ragionamento de Aretino, que establece las mismas premisas para las cortesanas.

El giro hacia el diálogo mimético que se produjo a mediados del Cinquecento está relacionado para Ordine con la crisis que sufre la sociedad cortesana durante la época. El caso de los diálogos se vuelve paradigmático: no se presta en ellos atención alguna a la circunstancia en que se desarrolla la conversación y los personajes no son ya representantes de un único estrato social, deviniendo modelos de comportamiento; sino que más bien representan una realidad pluricéntrica y dan muestra de los distintos ambientes (corte, academia, etc.), forjando y oponiendo distintos códigos y opiniones: «il corpus dialogico di Speroni trova propio nel confronto delle diverse opinione la sua principale peculiarità» (p. 27). Es ejemplo de este giro el conjunto de los diálogos de Speroni, y de manera igualmente relevante su idea del diálogo como obra abierta.

Ordine toma como autor paradigmático en la evolución del diálogo mimético finisecular a Giordano Bruno. Las obras del Nolano carecen de lugares y de personajes reales, pero sobre todo destacan por su concepción del lenguaje: «la frattura tra dimostrazione e argomentazione, tra scienze dell'uomo e scienze della natura, che aveva caractterizzato il dibattito teorico sul genere dialogo si ricompone: per Bruno tutto ciò che esiste parla un medesimo linguaggio; un linguaggio che esprime, attraverso l'unità dei saperi, la complessità di un universo infinito continuamente agitato dalla mutazione» (p. 29). Es evidente que la brevedad del trabajo de Ordine no permite un tratamiento exhaustivo de una materia tan extensa como la producción dialógica en Italia durante el Cinquecento, ahora bien, la línea evolutiva que traza como propuesta de trabajo será adoptada con mayores o menores modificaciones en la parte más relevante de estudios que en adelante se dediquen a este campo.

A finales de la década aparece el que sigue siendo el estudio canónico 
sobre la poética del diálogo en el Renacimiento, Writing the Scene of Speaking (1989) de Jon R. Snyder. La diferencia esencial de este trabajo con los reseñados anteriormente y con los siguientes que veremos es que sólo atiende a los tratados específicos sobre el género producidos entre 1561 (la fecha que erróneamente se atribuye al tratado de Sigonio) y 1585 (fecha de redacción del Dell'arte del dialogo de Torquato Tasso), a los que se suman dos textos tardíos Del dialogo (1628) de Giambattista Manso y el Trattato dello stile e del dialogo (1662) de Pietro Sforza Pallavicino. Snyder propone en su capítulo introductorio las categorías y los problemas planteados en las escasas y fragmentarias reflexiones acerca del diálogo de la antigüedad clásica, mientras que cada uno de los apartados siguientes se dedica, respectivamente, a Sigonio, Speroni y Tasso. En el último capítulo se ocupa de Manso y Pallavicino que, en su opinión, marcan la crisis y la decadencia del discurso italiano sobre el diálogo. El trabajo del americano destaca, entre otras cosas, por la relación que establece entre teoría literaria contemporánea y reflexión quinientista sobre el diálogo. Probablemente, este hecho determina el escaso énfasis que se hace en la circunstancia cultural, más allá de lo inexcusable, que rodea a la redacción de los tratados: el nacimiento de la teoría moderna de los géneros en la Italia de la época. La referencia a la impronta de la poética aristotélica no es suficiente, ni convincente, en los problemas particulares que atañen al género y sólo explica, por sí misma, parte del tratado de Sigonio o las observaciones de Castelvetro. En este sentido, Snyder deja en segundo plano el problema de adscripción del diálogo a la poética neoaristotélica, para profundizar en su carácter híbrido y en los problemas que le son propios como discurso literario.

Frente al estudio excesivamente formal, teórico, de Snyder, aparecen durante el mismo año diferentes trabajos que implican a la historia cultural del diálogo, que acabarán por culminar en The Renaissance Dialogue in Its Social and Political Context (1992) de Virginia Cox. Debe destacarse, en primer lugar, el recorrido de Peter Burke sobre el diálogo y la conversación renacentista. Su artículo «The Renaissance Dialogue» (1989) hace una propuesta que el propio Burke ha desarrollado en The Art of Conversation (1993) y The Fortunes of the Courtier (1995), esto es, no dejar únicamente el estudio de las manifestaciones del género a los críticos literarios y a los filólogos, sino comprender el hecho conversacional codificado y las producciones dialógicas renacentistas como marco para asentar estudios sobre el lenguaje, el comportamiento, el pensamiento o la lectura durante la época, por tanto, como plataforma idónea para la proliferación de estudios culturales. Las obras subsiguientes de Burke son, como la amplia mayoría de su producción, fuentes ineludibles para ampliar las miras del estudioso literario cuando se enfrenta a problemas tangenciales a su campo de estudio; sin embargo, se trata de una perspectiva que influye 
bien poco, e incluso desenfoca nuestro conocimiento de la verdadera naturaleza de los parámetros poéticos y retóricos en los que el diálogo y las ideas renacentistas en torno a él se generan, se nutren y se distancian.

El último estudio de importancia que cierra la década de los ochenta es La società del dialogo. Retorica e ideologia nella letteratura conviviale del Cinquecento (1989), de Raffaele Girardi. Sin pretenderlo, la obra del italiano es la contrapartida de los trabajos de Burke. Su monografía se orienta atendiendo a cuatro problemas que estructuran las partes de su estudio: Teorie ed apologie, Tipologie ed sperienze: il fantastico, Tipologie ed sperienze: la fede y Tipologie ed esperienze: la scienza. En estos cuatro apartados entran problemas relacionados tanto con la idea del diálogo durante el Cinquecento, como con la consideración general de la literatura durante el periodo. Así, la primera parte asienta desde su título la clara diferencia entre teorías poéticas y apologías de la obra de uno mismo, esto es, entre la construcción que la preceptiva literaria hace del diálogo frente a la defensa de una praxis determinada. No es de extrañar, por tanto, que esta parte se vea culminada por el análisis de la Apologia de Sperone Speroni, que supone la respuesta más clara a la "teoría" y una reflexión acerca del diálogo donde se conjugan experiencia poética y pensamiento artístico, creación y fundamentos teóricos.

El problema propuesto en las tres partes subsiguientes nace de la oposición entre verdad y ficción; entre la pragmática de la obra literaria y su función social y los elementos creativos que la hacen contradictoria frente a dicha realidad. En el fondo, son problemas en estrecha consonancia con las propuestas teóricas del periodo y establecen una relación precisa que permite ensanchar los parámetros de la teoría del género para analizar la società del dialogo, o la cultura conviviale quinientista. En el caso de lo fantástico, por ejemplo, Girardi toma a aquellos autores que no pueden ser adscritos con facilidad dentro de la cultura dialógica apoyada en la idea de cortesía - Gelli, Doni, Franco- y cuya característica primordial es la experimentación tanto en la forma como en los contenidos. Estos modelos heterodoxos se consideran, con toda justicia, resultado de la influencia de cultivadores cuatrocentistas del género como Galateo o Alberti, cuyas referencias corresponden estrictamente con aquellas que eran directamente rechazadas por el clasicismo vigente: el Plutarco de los Moralia o Luciano con sus diálogos. Precisamente el mayor acierto en la recuperación y consideración de estos autores es destacar cómo la tendencia a la deformidad - expuesta por Speroni en su Apologia - y la adopción de fórmulas conscientemente anticlasicistas revelan una corriente teórica subterránea que determina y define de un modo complementario las ideas sobre el diálogo que nos lega la Academia.

En esta línea marcada por Girardi: el sustento de una metodología y de una visión teórica mucho más compleja y rica del diálogo quinientista 
que la aportada por los estudios más relevantes hasta el momento, continúa con Il «libro animato»: teoria e scrittura del dialogo nel Cinquecento (1992), de Carla Forno. No debe entenderse, empero, el trabajo de Forno como un análisis socio-histórico o cultural del género, sino como un planteamiento de los parámetros teóricos y creativos que condicionan la variedad y la enorme cantidad de testimonios que nos ha legado el Renacimiento.

El análisis se caracteriza a lo largo de toda su escritura por el equilibrio mantenido en el análisis de reflexión y creación poéticas, y se divide en tres grandes núcleos que atienden —en ese orden - a la teoría del diálogo, a la función y descripción de los modelos clásicos y a su mixtura y a los puntos de contacto que el diálogo sostiene con otras especies poéticas. Si consideramos el carácter tardío de las reflexiones poéticas sobre el género, podría afirmarse que $I l$ «libro animato» comienza por los efectos que la varietà de manifestaciones produce en la teoría poética más que en las mismas causas. Desde una perspectiva moderna - aunque estrechamente ligada a los textos de la época- Forno se ocupa del concepto de imitatio, de las influencias e interdependencias vitales e intelectuales entre los preceptistas, del problema del estilo, de la oposición entre oralidad y escritura, del peso de la dialéctica en la concepción compositiva del diálogo; para desembocar en el análisis pormenorizado y aislado de cada uno de los preceptistas buscando el modo en que estructuran las relaciones entre autor, personaje y lector.

La segunda parte ejecuta un análisis a medio camino entre teoría y praxis, entre reflexión y creación poética, mediante el estudio de los modelos clásicos para el cultivo del género: el modelo socrático expresado a través del diálogo platónico en sus distintas vertientes; el modelo lucianesco, al que se vincula con gran acierto con el modelo plutarquiano; y el modelo ciceroniano, que se analiza desde las premisas y métodos constructivos retóricos que potencian su finalidad didáctica.

La última parte ahonda en el asunto de la teoría y la realidad genérica, atendiendo a su carácter mixto como justificación última de su variedad y cantidad: diálogo y texto dramático; diálogo y novela; diálogo y epístola; dialogicidad y monologismo junto a las fórmulas retóricas determinantes para la creación del ambiente y el cierre del texto son los aspectos más relevantes que ocupan a su autora.

El otro estudio aparecido durante el mismo año es Le dialogue (1992) de Suzanne Guellouz. La francesa se propone escribir un trabajo que aúne perspectiva diacrónica e interdisciplinar para lograr «l'élaboration d'une monographie du genre, c'est-à-dire une étude qui, tout en prenant en compte [...] le plus grand nombre possible de textes, mette en valeur ce qui peut être considéré comme permanent, autrement dit les éléments constitutifs de la forme, sa poétique» (p. 21). Un objetivo tan ambicioso: la definición de la forma dialógica a través de épocas y disciplinas, desborda con mu- 
cho las posibilidades de cualquier monografía si tenemos en cuenta el desarrollo de los estudios parciales sobre el diálogo. Es demasiado pronto para elaborar una visión cabal de las directrices que organizan este modo de expresión. Un ejemplo claro de esta imposibilidad es el perfil histórico que aporta Guellouz (pp. 167-250), que resulta no sólo inexacto, sino simple en exceso ante todo por lo que respecta al estudio del diálogo medieval y renacentista. De la misma manera, el ahondamiento en los principios definitorios de la poética del género (pp. 77-164) es llamativamente reduccionista y no repara en la gran cantidad de nexos y de implicaciones con otros géneros que el diálogo mantiene por su esencia misma.

1992 es también fecha de aparición para la monografía de Virginia Cox. Frente a estudios como los de Girardi y Forno, preocupados por dar respuesta a la clara predominio del diálogo como género literario durante el siglo XVI, Cox trata de justificarla desde la historia intelectual del periodo. La distinción de partida que ordena todo el texto de la americana corresponde con la división que establece en el segundo capítulo entre diálogos documentales y ficcionales. Mientras que los primeros se producen siguiendo el modelo platónico-ciceroniano y representan conversaciones entre personajes reales ambientadas en un marco concreto; los segundos, influidos por la praxis lucianesca, suponen un juego de fantasía en donde el manejo de las ideas por parte de los interlocutores, su presencia incluso como encarnación de las mismas, pesa más que el verismo histórico. Cox propone que durante el Cinquecento la primacía del diálogo documental pasa por Il Cortegiano de Castiglione y la historia del diálogo durante el siglo corresponde con la transición del diálogo documental abierto a la cerrazón y desarticulación de la forma dialógica. Los motivos para esta "involución" son varios y atañen al contexto histórico y cultural más que al estrictamente literario: la esclerotización de las cortes italianas como fuentes privilegiadas para la creación literaria, el peso de la Contrarreforma que afectó a la creación de diálogos que aportaran posturas heterodoxas, la influencia y desarrollo de la cultura escrita y visual frente a la oral que propició el asentamiento de la imprenta y la nueva concepción tanto del libro como de sus funciones fueron los factores de mayor peso, para Cox, en el cambio esencial de concepción del diálogo.

Las observaciones de mayor interés en The Renaissance Dialogue in its Social and Political Contexts son, quizás por eso mismo, las más discutibles. La primera de ellas es la consideración del diálogo desde la ejecución y la especulación teórica del siglo XVI; la segunda es el motor principal que impulsa a todo el estudio: explicar a qué se debe la diferencia entre el cultivo del género en la Italia de la época y el resto de países europeos. El primero es discutible porque no es necesario, ni aconsejable, buscar un vínculo —o establecerlo sin más - entre teoría poética y cultivo del género: en casos como los de Sigonio o Castelvetro no hay una co- 
rrespondencia entre una y otra y, en Speroni, su misma concepción teórica debe ser matizada cotejando sus reflexiones no sólo con su ejercicio literario, sino con la circunstancia intelectual y la presión institucional que ronda a la redacción del mismo, tal y como ya había explicado Jon R. Snyder.

La diferencia propuesta entre Italia y el resto de países europeos debe ser matizada, puesto que, aunque el ejercicio dialógico italiano no pueda ser igualado en tono y en variedad a algunos de los países europeos - lo cual es muy discutible, sobre todo si tomamos la medida a las distintas circunstancias histórico-culturales que condicionan la producción literaria en cada uno de ellos-, esto no supone que precisamente esas obras tan "diferenciadas" - Il Cortegiano, Gli Asolani, I Dialoghi de amore, etc.no fueran absorbidas y traducidas, e incluso imitadas, casi con inmediatez por España, Francia o Inglaterra. Esa influencia, y el carácter paneuropeo de las obras italianas - aunque, como bien precisa Cox, adoptadas y modificadas más o menos según la tradición literaria del país que las integraba en su "canon" moderno- es el aspecto más relevante para la comprensión del diálogo renacentista desde una perspectiva comparatista y no al contrario. Bien es cierto que Cox propone explicar la peculiaridad de Italia buscando las motivaciones para su cultivo exhaustivo en el diálogo neolatino cuatrocentista, pero justamente la influencia y la distribución de algunas obras de enorme peso literario no puede obviarse por el análisis cuantitativo de las obras "originales" de cada país; debe atenderse más bien a cómo aquéllas hallan y tantean un cauce expresivo y compositivo que es común en las potencias literarias europeas.

Durante el año siguiente, aparecen dos breves trabajos que tratan la poética del diálogo desde las dos perspectivas fundadas durante la década de los setenta. Theories of Dialogue de Donald Gilman insiste en el peso que la dialéctica tuvo para las percepciones quinientistas sobre el diálogo; mientras que Il «Giardino» delle parole. Itinerari di scrittura e modelli letterari nel dialogo cinquecentesco de Valerio Vianello, ahonda en la construcción esencialmente retórica del diálogo renacentista.

Theories of Dialogue tiene la clara voluntad de enfocar la poética del diálogo como un fenómeno europeo - francés e italiano- y para ello selecciona sólo los tratados de Sigonio y Tasso, y añade al "canon" de preceptistas el "préface" de Louis Le Caron a su diálogo La Claire (1551). Gilman pretende establecer con los textos seleccionados la evolución europea en la poética del diálogo: mientras que el texto de Le Caron busca justificar su misma práctica literaria y conceder al diálogo el estatuto de especie poética en la Francia de mediados del siglo XVI, el De dialogo liber puede leerse como una definición del mismo género que el francés había defendido diez años antes; dentro de esta línea evolutiva, el breve Discorso tassiano supone una amplificación de las limitaciones críticas que el mismo Sigonio le había impuesto a la forma: no sólo no se expurga la 
forma lucianesca, sino que la primacía del régimen de la enargeia como motor del género, y la ambivalencia misma del diálogo como género y modo tomada de los Poetices libri septem permite armonizar experimentación y convención genérica.

Gilman no atiende a los trabajos de Castelvetro y de Speroni porque su interés principal es ahondar en los vínculos existentes entre poética del diálogo, dialéctica y retórica. Para ello se concentra en el estatuto y en la sistematización que se concede a la argumentación en los tratados. El aserto común a Le Caron, Sigonio y Tasso — que el diálogo es la manifestación poética de la dialéctica - se va mostrando como una afirmación superficial: la idea de dialéctica que defienden sólo puede ser comprendida a partir del principio que orquesta toda la Retórica aristotélica, es decir, que la retórica es una contrapartida de esta. Lo interesante del trabajo de Gilman es precisamente cómo a partir del análisis de este aspecto es capaz de trazar una línea evolutiva que culmina con la primacía retórica en Tasso para la expansión de la poética a las variedades en que el género se manifiesta.

Il «giardino» delle parole parte del extremo opuesto al anterior trabajo, y frente a él, retoma el estudio conjunto de poética y praxis dialógica durante el siglo XVI. Tras un breve capítulo dedicado a la síntesis de las teorías poéticas de la segunda mitad del Cinquecento, Vianello trata de explorar las ideas que los diálogos más representativos de la primera mitad del siglo aportan sobre los modos de construcción dialógica. Esta parte comienza su andadura con Gli Asolani, de Pietro Bembo, que el italiano estudia mediante el recorrido de sus diferentes redacciones y el establecimiento de calas en sus más relevantes elementos constitutivos: su carácter literario-amoroso, apoyado en una referencia continua al Canzionere petrarquesco, su concepción por tanto de la obra como memoria o recorrido por los distintos estadios del proceso amoroso; su reflejo de la cultura cortesana y de la biografía literaria e intelectual de Bembo; la intertextualidad novelística con textos como el Decamerón, etcétera. Todo ello para demostrar no sólo como «lo scorrimento del progetto, come per altri illustri esempi dil primo Cinquecento, si misura su tempi lunghi, attraverso una preistoria complessa di ripensamenti e di riaggiustamenti, che accumulano stratificazioni culturali ed esistenziali per il tramite di fase preparatorie e di una rilettura delle esperienze personali» (p. 43); sino también, y principalmente, que estas fases de redacción ofrecen dos resultados bien distintos: uno fruto de las exigencias de una sociedad cortesana y sus códigos, tanto desde la filosofía (poesía) amorosa, como desde la presencia de Boccacio y la relación "natural" entre el amor y las letras; otro, el definitivo, donde se convierte en obra maestra del clasicismo, resultado de la búsqueda de su estatura universal.

Vianello considera que lo opuesto sucede en el Cortegiano de Castiglione, puesto que esta obra se concibe desde su misma redacción con una 
clara vocación de historicidad, de recreación de una conversación que ha tenido lugar, alejada en este sentido del tratado, de manera que deviene fijación literaria de una suma de valores, de un código, que rinde testimonio de una cultura asociada a un núcleo de poder y transmisión ideológica concretos y a un momento histórico determinado. El siguiente capítulo, que atiende a las Prose della volgar lingua de Bembo, sitúa a la obra como meta del proceso intelectual de su autor, como culminación de aquellos elementos que ya se habían esbozado en la redacción definitiva de Gli Asolani. La clave, resumiendo mucho, es para Vianello la eliminación de aspectos secundarios — cortesanos, amorosos - de la primera obra, para dirigir la segunda hacia el ideal de la constitución de una palabra, de un modelo de palabra que constituya el molde autorizado para la composición en vulgar de las futuras generaciones.

En los dos capítulos siguientes se establece una oposición a un nivel superior. Durante la decadencia de los centros culturales cortesanos a comienzos del segundo tercio del siglo, aparecen dos obras que Vianello compara: el Dialogo della volgar lingua, de Pierio Valeriano y el Dialogo delle lingue, de Sperone Speroni. La obra de Valeriano, frente a Bembo, propone una conjunción de las diversas opiniones en torno al problema de la lengua, recogiendo y dando primacía entre todas precisamente a aquellas que ya habían sido rechazadas por el veneciano, es decir, las más marcadamente cortesanas. Speroni, por el contrario, representa la transición del centro cultural aristocrático en el primer tercio de siglo al nuevo mundo proyectado a través de las sociedades civiles: las academias. El primado de la elocutio, de la retórica, sobre la filosofía humanista - inventio y dispositio - es el giro, para Vianello, hacia un saber difundido, abierto, en vulgar, en una sociedad urbana pluricéntrica y compleja, civil y problemática.

Il discorso labirintico del dialogo rinascimentale (1995) de Olga Zorzi Pugliese ahonda de nuevo en los conceptos retóricos aplicados a la constitución del género en el Cinquecento mediante el estudio de la noción speroniana de laberinticità; análoga, en realidad, a la varietà que coordinaba la monografía de Carla Forno. Hay en este trabajo algunas peculiaridades y hallazgos que merecen ser considerados. El principal aparece en su extensa introducción, donde se desarrolla por lo menudo el concepto de laberinticità speroniano, que posee una carga epistemológica en la que se hallan directamente implicadas algunas de las obras más relevantes del Quattrocento y del Cinquecento: «come il labirinto, il dialogo, con la sua struttura indiretta, non rivela esplicitamente il suo messaggio, ma lo nasconde negli intrichi del suo interno[...]. É evidente [en Speroni] la sua enfasi sul paradosso e sull'ironia e anche per il doppio senso linguistico, detto da lui, "anfibologico parlamento"» (p. 47). Esta realidad se hace coincidir con otro concepto fundamental en la poética speroniana, el gio- 
co, el juego, que deviene un elemento estructurante, o desestructurante dentro del diálogo, que elimina la persecución de la verdad como una de sus premisas y establece que la única verdad es, en realidad, una ficción retórica, el establecimiento de oposiciones, de escapes, de desviaciones que conducen al texto hacia temas tangenciales, secundarios, divergentes, lo que hace que el género sea concebido como la forma compositiva abierta por excelencia dentro de los distintos modelos a los que podía aferrarse el humanista.

De aquí, pasan a ser analizadas cuatro obras que ejemplifican este proceso y, de alguna manera, con este mismo análisis se matiza y se expande la línea evolutiva que había propuesto Ordine. El Ad Petrum Paulum Histrum dialogus, de Leonardo Bruni; el De professione religiosorum, de Lorenzo Valla; Gli Asolani, del Cardenal Bembo e Il Galateo, de Giovanni Della Casa son las calas que sirven a Pugliese para enfatizar la progresiva degeneración del molde y la clara tendencia monológica que da el tono de la última de las obras seleccionadas. La gran novedad del trabajo es haber asociado al primer humanismo la corriente que aparece "sistematizada" en la Apologia de Speroni. Haber considerado el diálogo del siglo XV como muestra de rechazo al escolasticismo no es algo, ciertamente, novedoso dentro de la crítica italiana; ahora bien, establecer dentro de esos mismos parámetros una línea cultural con los planteamientos poéticos de Speroni y afianzar el progresivo peso de la Contrarreforma como fuerza represiva y determinante en el desarrollo finisecular de la forma, convierte a este breve libro en una pieza importante para la comprensión del diálogo dentro de los parámetros culturales del último Humanismo.

En la línea de los trabajos que indagan la naturaleza retórica del diálogo, merece especial atención un estudio que aparece tres años después de Il discorso labirintico rinascimentale: Aptum. Retorica ed ermeneutica nel dialogo rinascimentale del primo Cinquecento (1998), de Annick Paternoster. El objeto de este libro magistral es, frente a aquellos que se encargan de ofrecer una visión comprehensiva de los tres modelos clásicos y su transmisión renacentista, la influencia del modelo ciceroniano en la configuración de los textos dialogados más importantes de la primera mitad del siglo XVI y en sus antecedentes más inmediatos del siglo anterior. Más aún, la tesis esencial consiste en la afirmación de que el estudio de la minutio y de la dissimulatio conduce a reconocer la función socializante del aptum y del decoro, no sólo para la retórica, a la que sirven en la contextualización del material tópico, sino para los dos géneros literarios que Paternoster toma en consideración —el diálogo y el tratado del comportamiento-, debido a que toman a la retórica como disciplina-guía para la competencia social por ellas presupuestas (p. 253).

A través de las páginas de este estudio el decoro va cobrando una relevancia cada vez mayor para la explicación del diálogo cuatrocentista y qui- 
nientista. En el centro de dos tendencias de ruptura o contradicción -el diálogo cuatrocentista y los diálogos de Speroni y Tasso- se encuentra el diálogo cortesano, en donde el decoro cobra toda su relevancia argumentativa: los personajes poseen un máximo de credibilidad en función de su representatividad histórica, su homogeneidad cultural y su competencia. Este hecho, que es válido tanto para Castiglione como para Bembo, e incluso para la inversión de Aretino, tiene en Sigonio a su principal preceptista: «con Sigonio approdiamo ad una concezione del dialogo in cui l'esemplarità del decor esterno deve arginare la molteplicità dei punti di vista generabile dal decor interno. La cornice del dialogo, invece di essere dispositivo per la formazione del "giudicio" civile del lettore, diventa infine risultato della deliberazione prudente avvenuta nella mente dell'autore» (p. 257).

En el caso de Speroni, al contrario, el diálogo se concibe como una forma construida sobre el concepto de varietas. Este concepto, al igual que el de libertas, condicionan por entero la defensa de la segunda parte de la Apologia dei dialoghi, en ella se pone de manifiesto que esa misma variedad, la inobservancia de personajes reputados, la representación de diversos estratos, sirve para preservar todo el campo de las opiniones sobre el significado social de los tópicos sometidos a discusión. En el caso del Discorso tassiano se produce, desde un punto de vista teórico, la síntesis y la contemplación de ambos tipos dialógicos mediante la división entre diálogos civiles o "de costumbres" y diálogos filosóficos. Esta evolución marcada por la autora, que toma en consideración todos los elementos constructivos retóricos que rondan a la forma — desde su doble naturaleza en la época, esto es, política (civil) y técnica (compositiva) - y su plasmación en los principales textos de la primera mitad del siglo XVI, deja dos preguntas abiertas, dos propuestas de trabajo que deben ser tomadas en seria consideración: la primera de ellas consiste en «uno studio della struttura retorica del dialogo cinquecentesco che rendesse conto del bivio metodologico che si verificò secondo noi nel momento in cui la struttura dialogica, la quale nel dialogo umanistico era nel contempo modello comportamentale e modello epistemico, perse la sua valenza epistemica e si fece illustrazione del solo modello comportamentale, quello cioè della corte» (p. 261). La segunda, más relevante a efectos literarios, y menos necesitada de realizarse al arrimo de los estudios culturales, «il primo [il dialogo cortegiano], grazia alla sua forte funzione modellizzante, non solo codifica un filone a sé comprendente dialoghi di comportamento, dialoghi di Corte, e di cui si potrebbero isolare le strutture retoriche, ma codifica anche la conversazione in altri generi limitrofi, soprattutto nella raccolta di novelle che nel Cinquecento molto spesso si rifà ad un immaginario cortigiano» (p. 262).

En los últimos años, han aparecido algunos estudios que vienen a completar muchos de los aspectos que ya hemos visto en los trabajos mencio- 
nados. Aquí recojo sólo algunos de los principales: Scritture al crocevia. Il dialogo letterario nei seccoli XV e XVI (1999) de Stephano Prandi; el conjunto de ensayos recogidos por Walter Geerts, Annick Paternoster y Franco Pignatti en Il Sapere delle parole. Studî sul dialogo latino e italiano del Rinascimento (2001); Le dialogue à la Renaissance (2001), de Anne Godard y el reciente Defining Dialogue. From Socrates to the Internet (2003), de Geoffrey Rockwell. Son cuatro trabajos de muy distinto corte. Tanto Prandi como Godard retoman la reconstrucción del fenómeno dialógico durante todo el siglo XVI, en el primer caso en el Cinquecento italiano y en el segundo intentando dar una visión paneuropea. Il sapere delle parole merecería por sí solo una reseña, puesto que recoge los últimos trabajos de autores tan esenciales para el estudio de la materia como Olga Zorzi Pugliese, Raffaele Girardi, Franco Pignatti, Sergio Bozzola, Annick Paternoster o Walter Geerts. Completamente desvinculado de la perspectiva tradicional se muestra el trabajo de Rockwell, e igualmente discutible, puesto que trata de analizar los elementos constitutivos del diálogo como género discursivo mediante la aplicación y el ensanchamiento de los principios teóricos contenidos en las obras de Sigonio, Speroni y Tasso.

Los estudios sobre poética y cultivo del diálogo en la Francia del siglo XVI son relativamente escasos al compararlos con Italia. De todo el corpus de diálogos franceses, los coloquios escolares fueron los más tempranos en recibir atención desde el ámbito académico, analizados por el estudio de Louis Massebieau: Les Colloques scolaires du seizième siècle et leurs auteurs (1480-1570), de 1878. Tras él, habrá que esperar prácticamente un siglo para que aparezcan dos estudios de enorme importancia sobre el diálogo en la Francia renacentista. El primero de ellos es el extenso e inconsistente Le monologue, le dialogue et la sottie (1976) de Jean-Claude Aubailly, ocupado en la evolución de los géneros dramáticos desde la Baja Edad Media hasta el siglo XVI, prestando especial atención al diálogo como un eje estructurador en la configuración de los géneros dramáticos más que como género literario específico. Durante el mismo año aparece el que pasa por ser el único análisis comprehensivo de la práctica dialógica quinientista en Francia: Le dialogue philosophique dans la littérature française du seizième siècle, de Mustapha Kemal Bénouis. Se trata, empero, de un estudio parcial que encamina sus hallazgos hacia la comparación de modelos y tendencias, pero que deja la sensación tras su lectura de no haber logrado su meta.

En la década de los ochenta comienzan a aparecer estudios que se preocupan por varios aspectos novedosos del diálogo renacentista. Cabe destacar dos publicaciones colectivas: Le dialogue au temps de la Renaissance (1984), bajo la dirección de M. T. Jones-Davies y Le diálogue (1985), coordinado por P. R. Léon y P. Perron. En la misma década se publica otro trabajo que merece ser tenido en cuenta, más que para el estudio del 
diálogo, para los vínculos culturales que este sostiene con la cultura convivial renacentista en la Francia del XVI: Des mets et des mots. Banquets et propos de table à la Renaissance (1987) de Michel Jeanneret. Junto a ellos, pueden considerarse la recopilación de trabajos editados por Colette H. Winn (1993) y donde se encuentran —además del extenso texto de Donald Gilman dedicado a la poética- importantes aportaciones al estudio del diálogo renacentista entre 1547 y 1630. La parte que la recopilación de Winn dejaba de lado fue cubierta por Ruxandra Vulcan en una trabajo que ha ido cobrando el estatuto de clásico: Savoir et rhétorique dans les dialogues français entre 1515 et 1550 (1996).

En los quince años comprendidos entre 1990 y 2005 la bibliografía francesa se ha movido en direcciones que recorren vías un tanto diversas de la bibliografía italiana, que ha tendido a esclerotizarse en la atención a ciertos modelos privilegiados. Por un lado, se ha comenzado a prestar la atención debida al diálogo medieval y, por otro, la poética del diálogo se ha extendido a otras latitudes. En el primer caso son fundamentales los estudios pioneros que durante las dos últimas décadas ha venido desarrollando Peter Von Moos, cuyo trabajo y propuestas siguen sin tener el eco que, me parece, merecen. El modo en que atiende a la cultura del diálogo durante la Edad Media - ocupándose de su cultivo, rudimentos teóricos y manifestaciones socioculturales- es tanto muestra de las relaciones conceptualmente peligrosas entre un género, el diálogo, y una manifestación cultural, la conversación, como de la habilidad de un historiador que comprende nuestras carencias para una comprensión cabal de las particularidades del diálogo en el medievo y que se enfrenta al complejo de inferioridad que sigue manteniendo con respecto a su cultivo por parte de los humanistas. El trabajo de Von Moos, publicado a lo largo de los años en revistas especializadas de difícil acceso, ha sido recogido en un volumen convenientemente titulado Entre histoire et littérature: communication et culture au Moyen Âge (2005).

La importancia que el diálogo medieval ha ido cobrando durante los últimos años se ha visto reflejada en ejemplos de investigación como los recogidos por Annelie Volgers y Claudio Zamagni (2003) o en la monografía de Carmen Cardelle de Hartmann (2007), entre muchos otros. Todo ello parece indicar que la revolución en la arena de la literatura comparada provendrá de una reconsideración del corpus medieval. Precisamente, dos libros de Olga Weijers sobre el género de la disputatio: La 'disputatio' à la Facultè des arts de Paris (1200-1350 environ). Esquisse d'une typologie (1995) у La 'disputatio' dans les Facultés des arts au Moyen Âge (2002) han servido de punto de arranque para estudios de largo alcance como el reciente Dialectique et littérature: Les avatars de la dispute entre Moyen Âge et Renaissance (2005) de Béatrice Périgot. El libro de Périgot realiza en el fondo una reducción a la inversa - convierte el gé- 
nero de la disputatio en central para la comprensión del diálogo-, y aunque ya hemos visto que es un procedimiento histórica y conceptualmente discutible, es pertinente para salvar el tradicional hiato entre Edad Media y Renacimiento. Quizás lo más aconsejable en este caso sería "armonizar" la propuesta de Périgot con las líneas que se han abierto en el estudio de los comentarios medievales de Cicerón, de cuyas posibilidades tan buena muestra ofrece el volumen recientemente editado por Virginia Cox y John O. Ward (2006).

Por lo que toca a la poética del diálogo, los principales estudios franceses se hallan, en realidad, en manos de un escaso número de críticos. Pueden citarse cuatro nombres para cubrir el recorrido y los intereses de los estudiosos sobre este particular: Michel Le Guern (1981: 141-148), Eva Kushner ([1972-1996] 2004), Véronique Montagne (2001: 477-509) y Véronique Zaercher (2001: 1331-1348). En el capítulo de Le dialogue rabelaisien. Le Tiers livre exemplaire (2000: 159-210) dedicado a la poética del diálogo se encontrará el mejor resumen disponible en francés sobre el tema. Frente al sobrevalorado trabajo de Le Guern, o a las a veces confusas aproximaciones de Kushner a la poética renacentista como lo que es, un campo de especulación concreto y delimitado durante el Renacimiento, Zaercher recorre la poética del diálogo italiano, para utilizar a posteriori el De optimo genere disputandi colloquendique (1551) de Simon de Vallambert. El tratado, recuperado en fechas recientes, revela la importancia de la tradición teórica ciceroniana aplicada a la poética del diálogo. Este movimiento no sólo permite desmarcarse de la pesada carga estructural - y nacional- de la poética neoaristotélica, sino que pone también de manifiesto la importancia de una línea teórica que arranca de las obras del Arpinate en las artes de la conversación y su poderosa influencia en las ideas teóricas en torno al diálogo y a la novela.

\section{REFERENCIAS BIBLIOGRÁFICAS}

ARMSTRONG, C. J. R. «The Dialectical Road to Truth: The Dialogue». En: SHARRAT, P. (ed.). French Renaissance Studies, 1540-1570. Humanism and the Encyclopedia. Edinburgh: Edinburgh University Press, 1976, pp. 36-51.

AUBAILLY, J.-C. Le monologue, le dialogue et la sottie. Essai sur quelques genres dramatiques de la fin du moyen âge et du début du XVIe siècle. Paris: Honoré Champion, 1976.

BATKIN, L. M. «Il dialogo». En: Gli umanisti italiani. Stile di vita e di pensiero. RomaBari: Editori Laterza, 1990, pp. 123-176.

BÉNOUIS, M. K. «La réforme ramiste et le genre du dialogue». Romance Notes. 1972, XIV: 1, pp. 155-161.

—. Le dialogue philosophique dans la littérature française du seizième siècle. The Hague - Paris: Mouton, 1976.

BURKE, P. «The Renaissance Dialogue». Renaissance Studies, 1989, III: 1, pp. 1-13. 
—. The Art of Conversation. Ihaca, NY: Cornell University Press, 1993.

- The Fortunes of the Courtier. Cambridge: Polity Press, 1995. Traducción castellana: Los avatares del Cortesano. Lecturas e interpretaciones de uno de los libros más influyentes del Renacimiento. Barcelona: Gedisa, 1998.

CARDELLE DE HARTMANN, C. Lateinische Dialogue 1200-1400. Literaturhistorische und Repertorium. Leiden - Boston: E. J. Brill, 2007.

COX, V. The Renaissance Dialogue. Literary Dialogue in Its Social and Political Contexts, Castiglione to Galileo. Cambridge: Cambridge University Press, 1992.

The Rhetoric of Cicero in Its Medieval and Early Renaissance commentary tradition. (Cox, V. y Ward, J. O. (eds.). Leiden - Boston: E. J. Brill, 2006

FERRONI, G. (ed.). Il dialogo. Scambi e passagi della parola. Palermo: Sellerio, 1985.

FORNO, C. Il "libro animatto": Teoria e scrittura del dialogo nel Cinquecento. Torino: Tirrenia Stampatori, 1992.

FRIEDLEIN, Roger. Der Dialog bei Ramon Llull: literarische Gestaltung als apologetische Strategie. Tübingen: M. Niemeyer, 2004.

- (ed.). El diálogo renacentista en la Península Ibérica - Der Renaissancedialog aud der Iberischen Halbinsel. Stuttgart: Franz Steiner Verlag, 2005.

GEERTS, W. Paternoster, A. y. Pignatti, F. (eds.). Il sapere delle parole. Studi sul dialogo latino e italiano del Rinascimento. Giornate di Studio, Anversa 21-22 febbraio 1997. Roma: Bulzoni, 2001.

GILMAN, D. «The Reconstruction of a Genre: Carolus Sigonius and the Theorization of Renaissance Dialogue». En: DALZELL: A.; FANTAZZI, Ch. y SCHOECK, R. J. (eds.). Acta Conventus Neo-Latini Torontoniensis. Proceedings of the Seventh International Congress of Neo-Latin Studies. Toronto, 8-13 August 1988. Binghamton-New York: Medieval \& Renaissance Texts \& Studies, 1991, pp. 345-355.

—. «Theories of Dialogue». En: WINN, C. H. (ed.). The Dialogue in Early Modern France. 1547-1630. Art and Argument. Washington: DC: The Catholic University of America Press, 1993, pp. 7-76.

GIRARDI, R. «Elegans imitatio et erudita: Sigonio e la teoria del dialogo». Giornale Storico della Letteratura Italiana. 1986, CLXII, (pp. 321-354.

—. La società del dialogo: retorica e ideologia nella letteratura conviviale del Cinquecento. Bari: Adriatica Editrice, 1989.

GUELlOUZ, S. Le dialogue. Paris: Presses Universitaires de France, 1992.

HEMPFER, K. W. Möglichkeiten des Dialogs. Struktur und Funktion eine literarischen Gattung zwischen Mittelalter und Renaissance in Italien. Stuttgart: Franz Steiner, 2002.

- (ed.). Poetik des Dialogs. Aktuelle Theorie und rinascimentales Selbstverständnis. Stuttgart: Franz Steiner, 2004.

—. Der Renaissance Dialog auf dem Iberischen Halbinsel. Stuttgart: Franz Steiner, 2005.

HIRZEL, R. Der dialogue. Ein Literarhistorischer Versuch. Leipzig: S. Hirzel, 1895. 2 vols

JEANNERET, Michel. Des mets et des mots. Banquets et propos de table à la Renaissance. Paris: José Corti, 1987.

JONES-DAVIES, M. T. (ed.). Le dialogue au temps de la Renaissance. Paris: Jean Touzot Libraire, 1984.

KUSHNEr, E. «Réflexions sur le dialogue en France au XVIe siècle». Revue de sciences humaines. 1972, XXVII, pp. 485-501.

—. «Renaissance Dialogue: Work of art or instrument of inquiry?». Zagadnie rodzajow literackich (Revue des genres littéraires). 1977, XXXIX, pp. 23-35.

—. «Le dialogue en France a la Renaissance: quelques critères génologiques». Revue Canadienne de Littérature Comparée. 1978, V, pp. 142-153.

—. «Le diálogue de 1590 à 1630: articulations et functions». En: LAFOND, J. y. STEGMAN, A. (eds.). L, automne de la Renaissance 1580-1630. XXIIe Colloque International d,Études Humanistes, Tours 2-13 juillet 1979. Paris: J. Vrin, 1981, pp. 149-161. 
- «Le rôle structurel du locus amoenus dans le dialogues de la Renaissance». Cahiers de l,Association Internationale des Études Françaises. 1982, XXXIV, pp. 141-153.

—. «Le dialogue en France de 1550 à 1560». En: JONES-DAVIES, M. T. (ed.). Le dialogue au temps de la Renaissance. Paris: Jean Touzot, 1984, pp. 151-167.

—. «Vers une poétique du dialogue de la Renaissance». En: VV. AA. Essays presented to C. M. Vajda on his 70th birthday. Szeged: J. A. Tudomànyegetem, 1983, pp. 131-136.

—. «Dialogue et verité: réflexions autour de Pontus de Tyard». En: CÉARD, J. (ed.). Études offertes à Jean Claude Margolin. Genéve: Droz, 1993, pp. 99-109.

—. «Epilogue: le dialogue des dialogues». En: WINN, C. H. (ed.). The Dialogue in Early Modern France, 1550-1630: Art and Argument. Washington: The Catholic University of America Press, 1993, pp. 259-283.

—. «The Renaissance Dialogue and Its Zero-Degree Fictionality». En: MIHAILESCU, C.A. y HAMARNEH, W. (eds.). Fiction Updated: Theories of Fictionality, Narratology, and Poetics. Toronto \& Buffalo: University of Toronto Press, 1996, pp. 165-172.

—. Le Dialogue à la Renaissance. Histoire et poétique. Genève: Droz, 2004.

LE GUERN, M. «Sur le genre du dialogue». En LAFOND, J. y STEGMAN, A. (eds.). L'automne de la Renaissance, 1580-1630. XXIIe Colloque International d'Études Humanistes. Tours, 1979, 2-13 juillet. Paris: J. Vrin, 1981, pp. 141-148.

MARSH, D. The Quattrocento Dialogue: Classical Tradition and Humanist Innovation. Cambridge, MA: Harvard University Press, 1980.

MASSEBIEAU, L. Les Colloques scolaires du seizième siècle et leurs auteurs (1480-1570). Paris: Bonhoure, 1878

MCCLELLAND, J. «Dialogue et rhétorique à la Renaissance». En: LÉON, P. R. y PERRON, P. (eds.). Le dialogue. Ottawa: Didier, 1985, pp. 157-164.

MERRILL, E. The Dialogue in English Literature. Yale: Yale University Press, 1911. Reimpresión en Archon Books, 1969.

MONTAGNE, V. «Jean Sturm \& Valentin Erythraeus, ou l'élaboration méthodique d'une topique dialectique». Bibliothèque d'Humanisme et Renaissance. 2001, LXIII: 3, pp. 477-509.

MULAS, L. «Funzioni degli essempi, funzioni del Cortegiano». En: OSSOLA, C. (ed.). La corte e Il Cortegiano. Roma: Bulzoni, 1980, pp. 97-117.

—. «La scrittura del dialogo. Teorie del dialogo tra Cinque e Seicento». En: CERINA, G.; LAVINO, C. y. MULAS, L. (eds.). Oralità e scrittura nel sistema letterario. Atti del Convegno Cagliari, 14-16 aprile 1980. Roma: Bulzoni, 1982, pp. 245-263.

ORDINE, N. «Il dialogo cinquecentesco tra diegesi e mimesi». Studi e problemi di critica testuale. 1988, XXVII, pp. 155-179. Publicado y revisado bajo título distinto: «Teoria e 'situazione' nel dialogo del Cinquecento italiano». En: BIGALLI, D. y CANZIANI, G. (coords.). Il dialogo filosofico nel '500 europeo. Atti del convegno internazionale di studi. Milano 28-30 maggio 1987. Milano: Franco Angelli, 1990, pp. 13-33.

—. «Préface. La théorie du dialogue au XVIe siècle». En Le Tasse, Discours su le dialogue. Vuilleumer, F. (trad.); Baldassarri, G. (notas). Paris: Les Belles Lettres, 1992, pp. 9-61.

PATERNOSTER, A. Aptum: retorica ed ermeneutica nel dialogo rinascimentale del primo Cinquecento. Roma: Bulzoni, 1998.

PÉRIGOT, B. «Le dialogue theorisé au XVIe siècle: émergence d'un genre entre dialectique et littérature». Loxias, 2004, IV.

- Dialectique et littérature: les avatars de la dispute entre Moyen Âge et Renaissance. Paris: Honoré Champion, 2005.

PIGNATTI, F. «I Dialoghi di Torquato Tasso e la morfologia del dialogo cortigiano rinascimentale». Studi Tassiani, 1988, XXXVI, pp. 7-43.

—. «Introduzione». En: SIGONIO, Carlo. De dialogo liber - Del dialogo. Pignatti, F. (ed.); Patrizi, G. (pref.). Roma: Bulzoni, 1993, pp. 13-108. 
—. «Il dialogo del Rinascimento. Rassegna della Critica». Giornale Storico della Letteratura Italiana. 1999, CLXXVI: 575, pp. 408-443.

PRANDI, S. Scritture al Crocevia. Il dialogo letterario nei secc. XV e XVI. Vercelli: Edizioni Mercurio, 1999.

ROCKWELL, G. Defining Dialogue: From Socrates to the Internet. New York: Prometheus Books, 2003.

SNYDER, J. R. Writing the scene of speaking: theories of the dialogue in the Late Renaissance. Stanford: Stanford University Press, 1989.

TATEO, F. «Il dialogo morale». En Tradizione e realtà nell'Umanesimo italiano. Bari: Dedalo Libri, 1967, pp. 291-390.

VASOLI, C. La dialettica e la retorica dell'Umanesimo. "Invenzione” e "Metodo" nella cultura del XV e XVI secolo. Milano: Feltrinelli, 1968.

VIANELLO, V. Il "giardino» delle parole. Itinerari di scrittura e modelli letterari nel dialogo cinquecentesco. Roma: Jouvence, 1993.

VOLGERS, A. y ZAMAGNI, C. (eds.). Erotapokriseis. Early Christian Question-andAnswer Literature in Context. Proceedings of the Utrecht Colloquium, 13-14 October 2003, Leuven, 2004

VON MOOS, P. I. Entre histoire et littérature: communication et culture au Moyen Âge. Firenze: SISMEL - Edizioni del galluzzo, 2005.

VULCAN, R. I. Savoir et rhétorique dans les dialogues français entre 1515 et 1550. Münster - Hamburg - Berlin - Wien - London - Zürich: LIT Verlag, 1996.

WEIJERS, O. La 'disputatio' à la Faculté des arts de Paris (1200-1350). Esquisse d'une typologie. Turnhout: Brepols, 1995.

—. La 'disputatio' dans les Facultés des arts au moyen âge. Turnhout: Brepols, 2002.

WILSON, K. J. Incomplete Fictions: The Formation of English Renaissance Dialogue. Washington, DC: The Catholic University of America Press, 1985.

WINN, C. H. (ed.). The Dialogue in Early Modern France. 1547-1630. Art and Argument. Washington, DC: The Catholic University of America Press, 1993.

WYSS MORIGI DI ROHRBACH, G. Contributto allo studio del dialogo all'epoca dell'Umanesimo e del Rinascimento. Monza: Scuola Tipografica Artigianelli, 1947.

ZAERCHER, V. Le dialogue rabelaisien. Le Tiers Livre exemplaire. Genève: Librairie Droz, 2000.

- - «Entre dispute et conciliation: strategies et figures du «consensus» dans le dialogue de la seconde moitié du XVIe siècle». Revue d'Histoire Littéraire de la France. 2001, CI 5, pp. 1331-348.

ZORZI PUGLIESE, O. «Speroni Sperone and the Labyrinthine Discourse of Renaissance Dialogue». En: HART, J. (ed.). Imagining Culture. Essays in Early Modern History and Literature. New York \& London: Garland Publishing, 1996, pp. 57-72, 200-1.

-. Il discorso labirintico del dialogo rinascimentali, Roma: Bulzoni, 1995.

Fecha de recepción: 14 de agosto de 2008

Fecha de aceptación: 10 de marzo de 2009 